\title{
ACCRETION DISK CORONA LINE EMISSION FROM X0614+091
}

\author{
D. J. Christian, ${ }^{1,2}$ N. E. White, ${ }^{3}$ and J. H. Swank ${ }^{1}$ \\ Laboratory for High Energy Astrophysics, NASA/Goddard Space Flight Center, Greenbelt, MD 20771 \\ Received 1993 May 13; accepted 1993 August 27
}

\begin{abstract}
The low-mass X-ray binary X0614 + 091 was observed on 3 days in 1979 with the Einstein Observatory solid state spectrometer and the monitor proportional counter. During the observation with the highest measured flux, corresponding to an X-ray luminosity of $8 \times 10^{36}$ ergs s$^{-1}$ (in the $0.5-20 \mathrm{keV}$ band for an assumed distance of $5 \mathrm{kpc}$ ), significant low-energy emission was detected, centered at $0.77 \mathrm{keV}$, possibly due to line emission for $\mathrm{O}$ VII-O VIII and Fe XVII-Fe XIX. The other observations, which were at fluxes lower by a factor of 2 , are consistent with the presence of the emission feature. The equivalent width of the feature, $37 \pm 6 \mathrm{eV}$, is of the same order as equivalent widths previously reported for more luminous low-mass X-ray binaries using grating spectrometer data. The soft X-ray lines could be emitted by gas expected to arise in an accretion disk corona excited by the central source. But to explain the observed feature, most of the corona needs to contribute, or other sources of emission are required.
\end{abstract}

Subject headings: accretion, accretion disks - binaries: close - stars: individual (X0614 + 091) stars: coronae - X-rays: stars

\section{INTRODUCTION}

The moderately bright X-ray source X0614+091 (Bradt \& McClintock 1983) is a low-mass X-ray binary (LMXB) system, based on its identification with a faint $(B \approx 18.8)$ blue star, V1055 Ori (Davidsen et al. 1974). The X-ray flux is variable by a factor of 2 on a timescale of hours, showing "Sco X-1 like" behavior, i.e., a correlation between spectral hardness and intensity (Mason et al. 1976). Recent CCD photometry found a 10 day modulation, which appears unlikely to be orbital because there is no evidence for a companion's contribution to the optical spectra (Machin et al. 1991). There was also no confirmation of a 5.2 day period previously proposed on the basis of Ariel V X-ray data (Marshall \& Millit 1981). During 14 hr of simultaneous observations, optical and EXOSAT X-ray fluxes were roughly anticorrelated as they changed by $20 \%$. An X-ray burst was discovered by Swank et al. (1978) from within $4^{\circ}$ of $\mathrm{X} 0614+09$ and most likely came from this source. Equating the peak burst flux to the Eddington limit for hydrogen on a $1.4 M_{\odot}$ star gave a distance of $\approx 5 \mathrm{kpc}$, consistent with the estimate of 4-8 kpc by Davidsen et al. (1974). This gave a range of $1-4 \times 10^{36} \mathrm{ergs} \mathrm{s}^{-1}$ for the $2-10 \mathrm{keV}$ luminosity of the persistent emission, at the lower end of the range seen from bright LMXB. The WATCH Monitor experiment on GRANAT recently found a burst from within $1^{\circ}$ of $\mathrm{X} 0614+09$ (Brandt et al. 1992), strengthening the conclusion that the source has occasional bursts. The flux was observed only above $6 \mathrm{keV}$, but suggested a peak burst flux 4 times larger than observed by OSO 8 . Brandt et al. (1992) point out that the source can only be as far as $3 \mathrm{kpc}$ if this larger luminosity is identified with that of the Eddington limit for helium (twice that for hydrogen).

Higher resolution instruments, the objective grating spectrometer (OGS) on board the Einstein Observatory (Giaconni et al. 1979) and the transmission grating spectrometer (TGS) on $E X O S A T$, have detected low-energy line emission in the

\footnotetext{
1 Code 666, NASA/GSFC, Greenbelt, MD 20771.

${ }^{2}$ Dept. of Astronomy, University of Maryland, College Park, MD 20742

${ }^{3}$ Code 668, Goddard Space Flight Center, Greenbelt, MD 20771.
}

0.3-1.5 keV bandpass from a number of other LMXB (Vrtilek et al. 1991; Kahn, Seward, \& Chlebowski 1984; Vrtilek et al. 1986a, b; Kallman 1989). This low-energy line emission may be from $L$ shell transitions of iron, and $K$ shell transitions of oxygen and nitrogen (Vrtilek et al. 1991). The location of this emission is unclear, although radiative recombination in an accretion disk corona has seemed likely (Kahn et al. 1984; Kallman, Vrtilek, \& Kahn 1989).

In this paper we report a strong low-energy feature in the SSS spectrum of the X0614+091. In $\S 2$ we discuss the observations which were taken simultaneously with the SSS and the MPC, the data, and the analysis of the spectra. We describe the results of fitting models to the SSS spectra and to the SSS and MPC spectra simultaneously. For a range of continuum models, the spectra establish an emission feature at about 0.77 $\mathrm{keV}$ and suggest a possible absorption edge near $0.87 \mathrm{keV}$. In $\S 3$ we discuss the physical implication of the results.

\section{OBSERVATIONS AND ANALYSIS}

$\mathrm{X} 0614+091$ was observed several times in 1979 by the Einstein Observatory's solid state spectrometer (SSS, $0.5-4.5 \mathrm{keV}$ ) and monitor proportional counter (MPC, 1.2-20.0 keV) (Giacconi et al. 1979). Table 1 gives a $\log$ of the observations. The SSS is described in Joyce et al. (1978). For X0614+091, the SSS data included rates (counts per $10 \mathrm{~ms}$ ) between $0.4 \mathrm{keV}$ and $4.5 \mathrm{keV}$ and pulse heights of single events to a channel width of $45 \mathrm{eV}$ with energy resolution of $160 \mathrm{eV}$. The MPC is described in Gaillardetz et al. (1978). The MPC data included counts per $2.56 \mathrm{~s}$ in eight pulse height channels from 1.2 to 20 $\mathrm{keV}$ with a resolution of $21 \%$ at $6 \mathrm{keV}$.

The MPC count rates show factor of 2 variations between observations, similar to the range seen by other X-ray experiments. For the first observation on 1979 March 30 the MPC count rate was 30 counts $s^{-1}$. On 1979 September 19 the source was brighter, giving 45 MPC counts $s^{-1}$ and 10 hours later 60 MPC counts $\mathrm{s}^{-1}$. An observation on 1979 October 2 gave a similar high-intensity level of $62 \mathrm{MPC}$ counts $\mathrm{s}^{-1}$

The SSS count rates cannot be interpreted directly in terms of source intensity variation because of a time dependence of 
TABLE 1

Einstein SSS AND MPC OBSERVATIONS OF X0614+091

\begin{tabular}{|c|c|c|c|c|c|c|c|c|c|}
\hline \multirow[b]{3}{*}{ TARGET } & \multicolumn{3}{|c|}{ OBSERVATION DATE } & \multicolumn{2}{|c|}{ SSS REAL TIME MOdE } & \multirow[b]{3}{*}{ QUANTITY } & \multirow[b]{3}{*}{$\left\langle e^{-\tau}\right\rangle$} & \multirow{2}{*}{\multicolumn{2}{|c|}{ MPC }} \\
\hline & Start Day & & Start Day & PHA & $I_{C E}^{d}$ & & & & \\
\hline & (1979) & Seconds & (1978) & Counts $\mathrm{s}^{-1 \mathrm{~b}}$ & Live Time $^{c}$ & & & Counts s ${ }^{-1 e}$ & Live Time ${ }^{c}$ \\
\hline 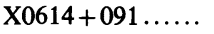 & Mar 30 & 35744 & 454.41 & 7.3 & 7232 & High & 0.23 & 30.5 & 8504 \\
\hline X0614+091...... & Sep 19 & 23490 & 627.27 & 17.6 & 1165 & Medium & 0.71 & 44.6 & 1610 \\
\hline X0614+091...... & Sep 19 & 63262 & 627.73 & 23.2 & 545 & Medium & 0.69 & 60.5 & 896 \\
\hline $\mathrm{X} 0614+091 \ldots \ldots$ & Oct 2 & 71213 & 640.82 & 28.3 & 3994 & Low & 0.96 & 62.2 & 1487 \\
\hline
\end{tabular}

a In the "real time mode," the dead time was $1-12 \mathrm{~ms}$ for pulse height information.

b PHA SSS channels 17-95 (0.61-4.25 keV).

c Good observation time, in seconds, satisfying constraints on background. The data selection criteria for the SSS and the MPC differ.

d Qualitative measure and the reduction in PHA counts $\mathrm{cm}^{-2} \mathrm{~s}^{-1} \mathrm{keV}^{-1}$ at $1 \mathrm{keV}$ due to ice absorption (see text).

' Channels 1-8(1.2-20 keV).

an ice deposit on the detector (Holt et al. 1979). The effect can be taken into account when the spectra are modeled by including the energy dependence of the X-ray absorption by the ice. When this is done the differences between the source fluxes seen by the SSS are comparable to the time variations of the MPC. The source showed $20 \%$ variations of intensity on time scales of $1000 \mathrm{~s}$, but on shorter time scales of 2.56-81.96 s, variability was less than $3 \%$ in the SSS energy bands $0.5-1.2$ $\mathrm{keV}, 1.2-3.5 \mathrm{keV}$, and $0.5-4.5 \mathrm{keV}$, and in the MPC bands $1.2-3.5 \mathrm{keV}, 3.5-10 \mathrm{keV}$, and 10-20 keV. Spectra accumulated for each observation in Table 1 represent a state of the source and do not average over intensity variations.

For the SSS spectral analysis we used the background from off source observations, including a correction for a variable particle component. We used the most recent calibration of the SSS response (Christian et al. 1992). The calibration includes a model for the accumulation of ice which was based on observations of the Crab Nebula made in March and September of 1979. The September Crab observations were made just before and just after the two September observations of X0614+091 and within a day of the October observation. The March observations of the Crab and X0614+091 were 5 days apart. The Crab observations are therefore especially good calibration references for the X0614+091 observations. This minimizes the uncertainties in the ice model.

As shown in Table 1, the attenuation due to ice was minimal for the October observation, modest for the September observations, and large for the March observation. In October the source was also the brightest, and these data give the clearest view of the low-energy spectrum.

Simultaneous MPC spectral data were obtained for each observing interval. Background for each satellite position was subtracted (Arnaud \& Vrtilek 1989). The same MPC response matrix was used that was generated recently for a spectral survey of 50 low-mass X-ray binaries (Christian 1993). It includes adjustments to the MPC model parameters to give consistency between the SSS and the MPC for sources with high column densities, e.g., GX 5-1. Without these adjustments, the MPC appeared to require low-energy flux $20 \%$ larger than required by the SSS. The adjusted response reproduces fits for sources with relatively low column densities, e.g., the Crab Nebula and clusters of galaxies.

For all but one of the X0614+091 spectra the joint MPC and SSS fits gave good agreement in the region of overlap $(1.2-4.5 \mathrm{keV})$. For the September observations of X0614+091, the MPC low-energy channels are $10 \%$ to $15 \%$ low with respect to the model fits to the simultaneous SSS and MPC data. Differences of this order are also seen for some observations of other sources for which the MPC rates are less than about $50 \mathrm{~s}^{-1}$. There is probably not a unique cause for such discrepancies. While the problem is small, only the MPC data for the October observation are used for this paper.

The October SSS data alone and the SSS data together with the MPC data were fitted to several models of continuum emission plus line emission, absorbed by a column density of interstellar gas with cosmic abundances (Morris \& McCammon 1983). The continuum models tested included thermal bremsstrahlung (TB), power-law (PL), blackbody (BB), and multicolor disks assuming blackbody emission (DBB) or modified blackbody emission (DMBB) by an $\alpha$-disk (Shakura \& Sunyaev 1973). Proportional counter spectra of X0614+091 have previously been approximated by thermal bremsstrahlung models with $k T \approx 4-6 \mathrm{keV}$. The best fits among the simple models were obtained for the unsaturated Comptonization approximation (USC), $d n / d E \propto E^{-\Gamma} \exp (-E / k T)$. The additional parameter $\Gamma$ allows it to approximate the emission from plasma of a range of optical depths and it successfully describes data from many sources (White, Peacock, \& Taylor 1985; Christian 1993).

The spectral fits to the October data for 82 SSS channels between 0.6 and $4.25 \mathrm{keV}$ were inconsistent with smooth continua such as a PL, TB, BB, USC, or disk emission $\left(\chi^{2}>300\right.$ for 79 per degree of freedom). An acceptable $\chi^{2}$ per degree of freedom (78/74) could be obtained including a lowtemperature blackbody component (BB), a narrow emission line (NL), and an absorption edge (ED). For the BB and NL components, reductions in $\chi^{2}$ were large, while the edge is barely significant. The line feature could also have breadth (FWHM $\sim 140 \mathrm{eV}$ ). The SSS spectrum is shown in Figure 1, together with the best fit, and the residuals relative to the best fits of three of the models: $\mathrm{PL}+\mathrm{BB}+\mathrm{NL}+\mathrm{ED}(b)$, $\mathrm{PL}+\mathrm{BB}+\mathrm{NL}(c)$, and $\mathrm{PL}+\mathrm{BB}(d)$. A complex of lines could act as a broadened feature. However, the combinations characteristic of collisional ionization equilibrium (as implemented in the Raymond \& Smith model of version 8 of the XSPEC Spectral Analysis package of the High Energy Astrophysics Science Archive Research Center at Goddard Space Flight Center) were inconsistent with the data.

The fitting procedure was repeated including the eight channels of MPC data. The results and parameters are shown in Table 2. The unsaturated Comptonization model plus blackbody (USC + BB) gave the best $\chi^{2}$ among the various continuum models tried. Because the underlying continuum is better constrained, the equivalent hydrogen absorbing column 


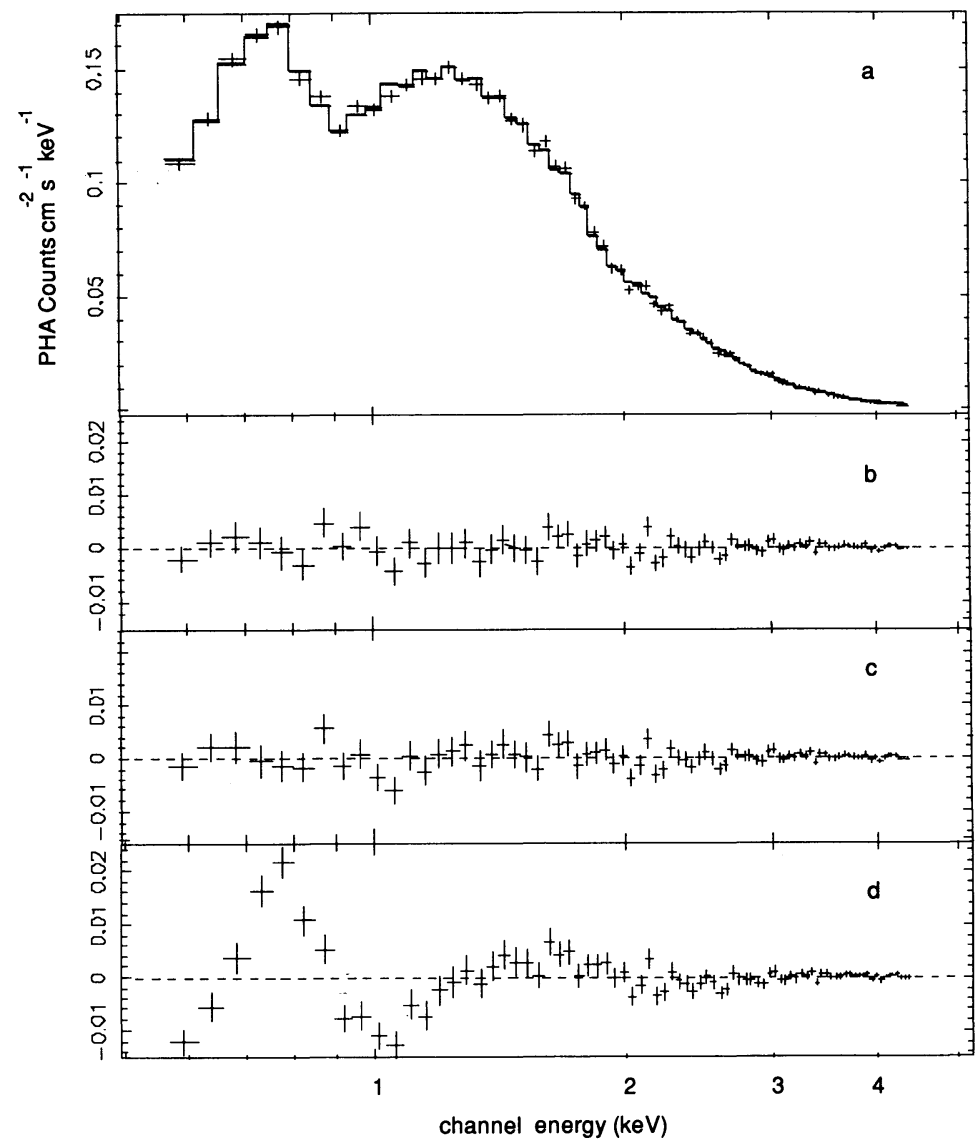

FIG. 1. - SSS spectral fits to the 1979 October 2 observation. (a) The data and best-fit PL + BB + NL + ED model. (b) The residuals of the data relative to the best-fit model. (c) The residuals relative to the best fit PL + BB + NL model. (d) The residuals relative to the best-fit PL + BB model.

density should be more accurate for the $0.5-20 \mathrm{keV}$ range of the SSS + MPC than for the SSS alone. The models $\mathrm{USC}+\mathrm{BB}+\mathrm{NL}+\mathrm{ED}, \quad \mathrm{TB}+\mathrm{BB}+\mathrm{NL}+\mathrm{ED}, \quad$ and $\mathrm{DMBB}+\mathrm{BB}+\mathrm{NL}+\mathrm{ED}$ give $N_{\mathrm{H}}=1.8 \pm 0.5 \times 10^{21} \mathrm{~cm}^{-2}$.

The equivalent width (EW) of the line, if narrow, was $37 \pm 6$ $\mathrm{eV}$, where the error is the variance for different models (Table 2). Figure 2 shows the best fit $(a)$ and residuals $(b)$ for both detectors for the model USC + BB + NL + ED. The edge gives a reduction in $\chi^{2}$ of 13 . The $1 \sigma$ errors on the edge energy were $0.85-0.92 \mathrm{keV}$.

There was more low-energy attenuation due to ice on the SSS detector during the September and March observations, which reduced the sensitivity to the low-energy line emission detected in October. Table 3 compares the fit parameters and $\chi^{2}$ for fits to the SSS spectra for each observation. A power-law continuum gave a good fit to the spectra of the SSS alone, but again only if a line feature was included. Figure 3 shows the residuals with respect to the continua of the best fits for the first three SSS observations. The emission line was consistent with being present in all observations, with EWs the same within a factor of 2 . Best fit EWs were $22 \pm 15 \mathrm{eV}$ for the low state in March, $34 \pm 11 \mathrm{eV}$ and $64 \pm 38 \mathrm{eV}$ for the two September observations, and $40 \pm 5 \mathrm{eV}$ for the October observation. From the October results, the model dependence of the EW is about $\pm 6 \mathrm{eV}$. For the early observations, there is no positive evidence for the edge, but the optical depth of $0.1-0.2$ giving the best fit for the October data is consistent with being present in all observations. There were no significant differ- ences in column densities of cold absorbing gas for the observations. (The model dependence of $N_{\mathbf{H}}$ in Table 2 is similar to the variation between observations in Table 3.)

\section{DISCUSSION}

The SSS and MPC spectra of X0614+091 establish several emission components that probably correspond to different regions in the system. While the continuum does not constrain a unique model, the variety of acceptable choices all imply the presence of a line emission feature.

Interpretation of the emission components depends on the assumed distance of the source. The equivalent hydrogen column density of absorbing gas, $(1.8 \pm 0.5) \times 10^{21} \mathrm{~cm}^{-2}$, required to fit the spectra, is consistent with reddening through the $1.5 \mathrm{kpc}$ of hydrogen layer in the direction of the source and with an upper limit on an interstellar line (Machin et al. 1990). Bursts from some sources have shown peak fluxes with a range of peak luminosities which may be less than or higher (e.g., van Paradijs et al. 1990) than either the hydrogen or helium Eddington limits. The conclusion from the burst observed by WATCH, that the source could be closer than previously estimated as close as $2 \mathrm{kpc}$, was based on a blackbody spectral assumption not verifiable from the data itself. We assume the distance is $5 \pm 3 \mathrm{kpc}$. Then the total flux for the October observation, $2.9 \times 10^{-9} \mathrm{ergs} \mathrm{cm}^{-2} \mathrm{~s}^{-1}$, corresponds to an X-ray luminosity of $8.7(+13,-8) \times 10^{36} \mathrm{ergs} \mathrm{s}^{-1}$. A $0.3 \mathrm{keV}$ blackbody component was detected, which contributed $1.8(+3$, $-1.5) \times 10^{36} \mathrm{ergs} \mathrm{s}^{-1}, 20 \%$ of the total. The flux in the emis- 
TABLE 2

SPECTRAL FITS TO THE SSS PLUS MPC FOR THE 1979 OCTOBER 2 OBSERVATION

\begin{tabular}{|c|c|c|c|c|c|c|c|c|c|c|c|}
\hline Model & $\alpha / \Gamma^{\mathrm{a}}$ & $\begin{array}{c}k T \\
(\mathrm{keV})\end{array}$ & Norm $^{b}$ & $\begin{array}{l}k T_{\mathrm{BB}} \\
(\mathrm{keV})\end{array}$ & $N_{\mathrm{BB}}^{\mathrm{b}}$ & $\left(10^{21} \stackrel{N_{\mathrm{H}}}{\mathrm{cm}^{-2}}\right)$ & $\begin{array}{c}F_{L}^{\mathrm{c}} \\
\left(\mathrm{cm}^{-2} \mathrm{~s}^{-1}\right)\end{array}$ & $\begin{array}{c}E_{L}^{\mathrm{d}} \\
(\mathrm{keV})\end{array}$ & $\begin{array}{c}\mathrm{EQW}^{\mathrm{e}} \\
(\mathrm{eV})\end{array}$ & $\tau$ & $\chi^{2} / \mathrm{dof}$ \\
\hline (2) & 2.38 & & 0.60 & $\ldots$ & $\ldots$ & 3.00 & $\ldots$ & $\ldots$ & $\ldots$ & $\ldots$ & $363 / 87$ \\
\hline (n. & $\ldots$ & 2.87 & 0.32 & $\cdots$ & $\begin{array}{l}\cdots \\
\cdots\end{array}$ & 1.70 & $\cdots$ & $\cdots$ & $\begin{array}{l}\cdots \\
\cdots\end{array}$ & $\begin{array}{l}\cdots \\
\cdots\end{array}$ & $761 / 87$ \\
\hline 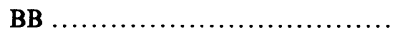 & $\ldots$ & 0.49 & 0.01 & $\ldots$ & $\ldots$ & 0.00 & $\ldots$ & $\ldots$ & $\ldots$ & $\ldots$ & $3528 / 87$ \\
\hline 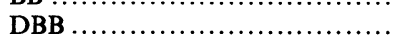 & $\cdots$ & $0.33^{\mathrm{f}}$ & 0.26 & $\cdots$ & $\cdots$ & 0.01 & $\cdots$ & $\cdots$ & $\ldots$ & $\ldots$ & $1851 / 87$ \\
\hline 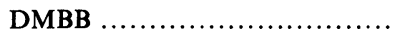 & $\ldots$ & $0.096^{\mathrm{f}}$ & 1.26 & $\ldots$ & $\ldots$ & 1.53 & $\ldots$ & $\ldots$ & $\ldots$ & $\ldots$ & 971/87 \\
\hline 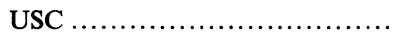 & 2.20 & 18.3 & 0.59 & & & 2.80 & $\ldots$ & $\ldots$ & $\ldots$ & $\ldots$ & $339 / 86$ \\
\hline $\mathrm{PL}+\mathrm{BB} \ldots \ldots \ldots \ldots \ldots \ldots \ldots \ldots \ldots \ldots \ldots \ldots \ldots \ldots$ & 2.33 & $\ldots$ & 0.50 & 0.45 & 0.001 & 2.80 & $\ldots$ & $\ldots$ & $\ldots$ & $\ldots$ & $343 / 85$ \\
\hline $\mathrm{DBB}+\mathrm{BB} \ldots \ldots \ldots \ldots \ldots \ldots \ldots \ldots \ldots \ldots \ldots$ & & & 0.92 & 1.60 & 0.008 & 1.36 & $\cdots$ & $\cdots$ & $\begin{array}{l}\cdots \\
\cdots\end{array}$ & $\cdots$ & $376 / 85$ \\
\hline $\mathrm{DMBB}+\mathrm{BB} \ldots \ldots \ldots \ldots \ldots \ldots \ldots$ & & $0.18^{\mathrm{f}}$ & 0.54 & 0.32 & 0.005 & 1.40 & & & & & $354 / 85$ \\
\hline TB + BB $\ldots \ldots \ldots \ldots \ldots \ldots \ldots \ldots \ldots \ldots \ldots \ldots \ldots$ & & 5.22 & 0.14 & 0.33 & 0.004 & 1.40 & $\ldots$ & $\ldots$ & $\ldots$ & $\ldots$ & $342 / 85$ \\
\hline $\mathrm{USC}+\mathrm{BB}+\mathrm{NL}^{\mathrm{g}} \ldots \ldots \ldots \ldots \ldots \ldots$ & 0.57 & 3.08 & 0.18 & 0.35 & 0.007 & 1.00 & 0.022 & 0.77 & 40 & $\ldots$ & $104 / 82$ \\
\hline $\mathrm{USC}+\mathrm{BB}+\mathrm{ED}^{\mathrm{h}} \ldots \ldots \ldots \ldots \ldots \ldots$ & 2.04 & 11.4 & 0.55 & 0.21 & 0.008 & 3.20 & & & & 0.32 & $141 / 84$ \\
\hline $\mathrm{PL}+\mathrm{BB}+\mathrm{NL}+\mathrm{ED} \ldots \ldots \ldots \ldots$ & 2.41 & $\ldots$ & 0.61 & 0.27 & 0.002 & 3.20 & 0.026 & 0.78 & 26 & 0.28 & $124 / 82$ \\
\hline $\mathrm{TB}+\mathrm{BB}+\mathrm{NL}+\mathrm{ED} \ldots \ldots \ldots \ldots$ & $\ldots$ & 5.2 & 0.15 & 0.30 & 0.006 & 2.30 & 0.023 & 0.77 & 38 & 0.23 & $95 / 82$ \\
\hline $\mathrm{DMBB}+\mathrm{BB}+\mathrm{NL}+\mathrm{ED} \ldots \ldots \ldots$ & & $0.18^{\mathrm{f}}$ & 0.52 & 0.31 & 0.006 & 1.83 & 0.023 & 0.77 & 37 & 0.18 & $93 / 82$ \\
\hline $\mathrm{USC}+\mathrm{BB}+\mathrm{ED}+\mathrm{NL} \ldots \ldots \ldots$ & $0.70_{-0.62}^{+0.56 i}$ & $3.30_{-1.1}^{+1.5}$ & $0.20_{-0.07}^{+0.10}$ & $0.33_{-0.03}^{+0.02}$ & $0.007_{-0.001}^{+0.001}$ & $1.28_{-0.3}^{+0.5}$ & $0.021_{-0.003}^{+0.004}$ & $0.77_{-0.01}^{+0.05}$ & $43_{-6}^{+8}$ & $0.14_{-0.07}^{+0.10}$ & $91 / 81$ \\
\hline
\end{tabular}

a $\alpha$ or $\Gamma$ in $d n / d E \propto E^{-\alpha}$ for PL model or $E^{-\Gamma} e^{-E / k T}$ for USC.

${ }^{b}$ Normalization for BB in units of $L_{39} /\left(D_{10}\right)^{2} . L_{39}$ is the luminosity in units of $10^{39} \mathrm{ergs} \mathrm{s}^{-1}$ and $D_{10}$ the distance in units of $10 \mathrm{kpc}$.

c Line flux in photons $\mathrm{cm}^{-2} \mathrm{~s}^{-1}$

d Energy of a narrow line.

' Line equivalent width.

${ }^{f}$ Mass accretion rate in terms of the Eddington limit $\left(2.4 \times 10^{18} \mathrm{ergs} \mathrm{s}^{-1}\right)$.

8 Narrow line.

${ }^{\text {h }}$ Absorption edge fixed at $0.87 \mathrm{keV}$.

Rms errors at $90 \%$ confidence. 


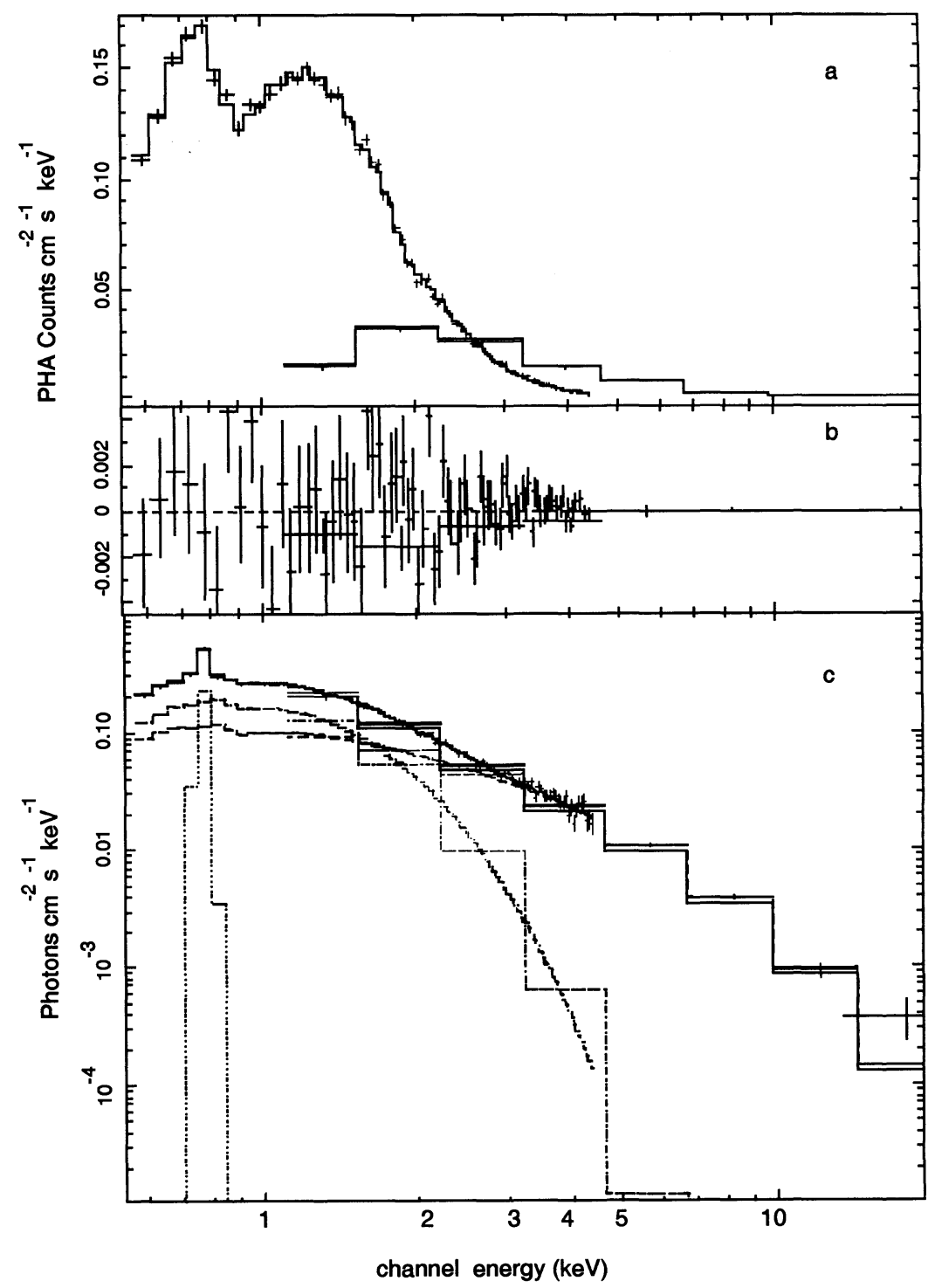

FIG. 2.-SSS + MPC spectral fit to the 1979 October 2 observation. (a) The data for the SSS from $0.5-4.5 \mathrm{keV}$ and the MPC from 1.2-20 keV with the fit to a $\mathrm{USC}+\mathrm{BB}+\mathrm{NL}+\mathrm{ED}$ model. $(b)$ The residuals relative to the fit. $(c)$ The model spectrum and components, with the estimated deconvolution of the data.

sion line feature, $7.8(+12,-7) \times 10^{43}$ photons $\mathrm{s}^{-1}$, corresponds to about $1.0(+1.6,-0.8) \times 10^{35} \mathrm{ergs} \mathrm{s}^{-1}$, or $1 \%$ of the total luminosity).

The blackbody projected area corresponds to a spherical radius of $30(d / 5 \mathrm{kpc})$ for the October observation. A radius as large as $30 \mathrm{~km}$ suggests the emission comes from the inner disk or the magnetosphere rather than the surface of a neutron star. If the source is at $2 \mathrm{kpc}$, then the blackbody radius is $12 \mathrm{~km}$ and could be a neutron star photosphere.

The possibly complex $0.77 \mathrm{keV}(16 \AA)$ feature, detected in all observations with an equivalent width of $\approx 30 \mathrm{eV}(0.6 \AA)$, is ascribable to transitions of O VII, O VIII, or Fe XVII-XIX (Shull 1979; Kallman \& McCray 1982). It has been argued for some Einstein OGS data of some LMXB (Cyg X-2, GX9+9, and $\mathrm{X} 1820-30$ ) that an emission feature observed near $0.77 \mathrm{keV}$ (16 $\AA$ ) must be due to $\mathrm{Fe} x \mathrm{xIII}$, rather than $\mathrm{O}$ VIII $\mathrm{Ly} \beta$, because the Ly $\alpha$ line at $0.65 \mathrm{keV}(19 \AA)$ is not observed (Liedahl et al. 1989, 1990). Vrtilek et al. (1991) point out that for several OGS observations of three bursters, X1636-536, X1735-444, and
$\mathrm{X} 1837+049$, emission lines at both $16 \AA$ and $19 \AA$ are detected, with the Ly $\alpha$ dominating, as would be the case for a gas in collisional ionization rather than photoionization equilibrium. The equivalent widths for these or nearby lines were 10-30 eV (0.1-0.3 $\AA$ ). The SSS data cannot rule out a contribution at the energy of the Ly $\alpha$ line. The three burst sources are similar to $\mathrm{X} 0614+091$ in several respects, in modest variablity of the nonburst X-ray flux, in the general nature of their X-ray continua, and in optical magnitude and color.

The physical picture of accretion through a disk onto a compact member of a binary system includes several possible sites of line emission, including the disk itself, coronal gas above the disk, the outer edge of the disk where the accretion stream from the companion meets the disk, a possible wind off the disk, and the part of the companion facing the X-ray source. The transitions that could contribute to the observed feature could be excited collisionally or in recombination on ions photoionized by the central X-ray source. An accretion disk corona is expected, in which lines are produced by the gas 
TABLE 3

COMPARISON OF SSS OBSERVATIONS

\begin{tabular}{|c|c|c|c|c|c|c|c|c|c|c|c|c|c|c|}
\hline \multirow[b]{2}{*}{ OBSERVATION } & \multirow[b]{2}{*}{ MODEL } & \multicolumn{2}{|c|}{ Power Law } & \multicolumn{2}{|c|}{ BLACKBODY } & \multirow[b]{2}{*}{$\underset{\left(10^{21} \mathrm{~cm}^{-2}\right)}{N_{\mathrm{H}}}$} & \multirow[b]{2}{*}{$\begin{array}{c}F_{L}^{\mathrm{c}} \\
\left(\mathrm{cm}^{-2} \mathrm{~s}^{-1}\right)\end{array}$} & \multirow[b]{2}{*}{$\begin{array}{c}E_{L}^{\mathrm{d}} \\
(\mathrm{keV})\end{array}$} & \multirow[b]{2}{*}{$\begin{array}{c}E Q W^{e} \\
(\mathrm{eV})\end{array}$} & \multirow[b]{2}{*}{$\tau$} & \multirow[b]{2}{*}{$\chi^{2} /$ dof } & \multicolumn{3}{|c|}{ FLUX $^{f}$} \\
\hline & & $\alpha$ & Norm $^{a}$ & $\begin{array}{l}k T_{\mathrm{BB}} \\
(\mathrm{keV})\end{array}$ & $N_{\mathrm{BB}}^{\mathrm{b}}$ & & & & & & & $\begin{array}{l}0.5-4.5 \\
(\mathrm{keV})\end{array}$ & $\begin{array}{c}2.0-10 \\
(\mathrm{keV})\end{array}$ & $\begin{array}{c}0.5-20 \\
(\mathrm{keV})\end{array}$ \\
\hline Mar $30 \ldots \ldots \ldots \ldots$ & PL & 2.15 & 0.19 & $\ldots$ & $\ldots$ & 3.29 & & & & $\ldots$ & $159 / 79$ & & & \\
\hline \multirow{2}{*}{$\begin{array}{r}454.41 \ldots \ldots \ldots \ldots \\
. \ldots \ldots \ldots \ldots\end{array}$} & $\mathrm{PL}+\mathrm{NL}^{\mathrm{g}}$ & 2.20 & 0.20 & & & 3.67 & 0.007 & 0.72 & 41 & & $139 / 77$ & & & \\
\hline & $\mathrm{PL}+\mathrm{BB}+\mathrm{NL}+E D^{\mathrm{h}}$ & 2.46 & 0.19 & 0.72 & 0.0012 & 3.67 & 0.010 & 0.73 & 22 & 0.05 & $121 / 74$ & 0.70 & 0.34 & 0.9 \\
\hline $\operatorname{Sep} 19 \ldots \ldots \ldots \ldots \ldots$ & PL & 2.18 & 0.28 & $\ldots$ & $\ldots$ & 2.70 & & & & $\ldots$ & $89 / 79$ & & & \\
\hline \multirow{2}{*}{$627.27 \ldots \ldots \ldots \ldots$} & $\mathrm{PL}+\mathrm{NL}$ & 2.23 & 0.29 & & $\ldots$ & 3.10 & 0.026 & 0.74 & 44 & & $60 / 77$ & & & \\
\hline & $\mathrm{PL}+\mathrm{BB}+\mathrm{NL}+\mathrm{ED}$ & 1.91 & 0.19 & 0.31 & 0.0019 & 2.40 & 0.014 & 0.76 & 34 & 0.16 & $52 / 74$ & 0.84 & 0.58 & 1.4 \\
\hline \multirow{4}{*}{$\begin{array}{l}\text { Sep } 19 \\
\quad 677.73 \ldots \ldots \ldots \ldots \ldots \\
\end{array}$} & PL & 2.34 & 0.53 & $\ldots$ & $\ldots$ & 3.50 & & & & $\ldots$ & $157 / 79$ & & & \\
\hline & $P L+N L$ & 2.50 & 0.63 & $\cdots$ & $\ldots$ & 4.50 & 0.14 & 0.73 & 103 & $\ldots$ & $119 / 77$ & & & \\
\hline & $\mathrm{PL}+\mathrm{BB}+\mathrm{NL}$ & 1.76 & 0.21 & 0.38 & 0.0054 & 2.50 & 0.050 & 0.74 & 88 & $\ldots$ & $112 / 75$ & & & \\
\hline & $\mathrm{PL}+\mathrm{BB}+\mathrm{NL}+\mathrm{ED}$ & 1.91 & 0.28 & 0.34 & 0.0052 & 2.80 & 0.044 & 0.75 & 64 & 0.23 & $109 / 74$ & 1.40 & 0.88 & 2.2 \\
\hline \multirow{2}{*}{$\begin{array}{l}\text { Oct } 2 \\
640.82\end{array}$} & & & & & & & & & & & & & & \\
\hline & $\mathrm{PL}+\mathrm{BB}+\mathrm{NL}+\mathrm{ED}$ & 1.62 & 0.25 & 0.33 & 0.006 & 1.90 & 0.026 & 0.77 & 40 & 0.14 & $78 / 74$ & 1.51 & 1.22 & 2.9 \\
\hline
\end{tabular}

b Normalization for $\mathrm{BB}$ in units of $L_{39} /\left(D_{10}\right)^{2} . L_{39}$ is the luminosity in units of $10^{39} \mathrm{ergs} \mathrm{s}^{-1}$ and $D_{10}$ the distance in units of $10 \mathrm{kpc}$.

c Line flux in photons $\mathrm{cm}^{-2} \mathrm{~s}^{-1}$.

d Energy of a narrow line.

e Line equivalent width.

Unabsorbed model flux in energy range given in units of $10^{-9} \mathrm{ergs}^{-2} \mathrm{~cm}^{-1}$

U Narrow line.

h Absorption edge fixed at $0.87 \mathrm{keV}$. 


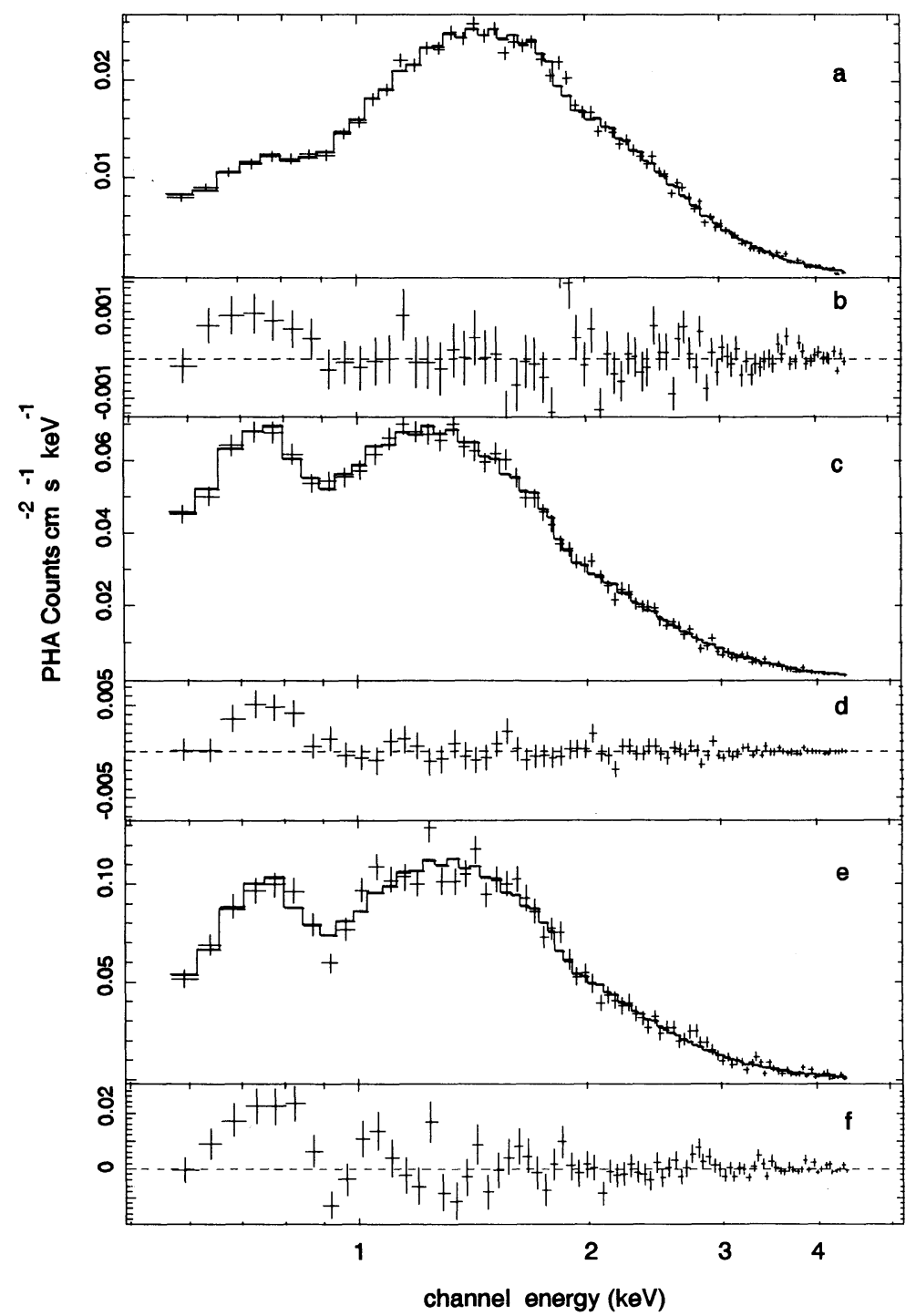

FIG. 3.-SSS spectra for 1979 March 30 and two observations on 1979 September 19. (a) The best-fit model with respect to the continuum plus line, and residuals with respect to only the continuum, of the best fit for the 1979 March 30 observation. (b) The same for the first observation on 1979 September 19. (c) The same for the second observation on 1979 September 19.

photoionized by the central X-ray source, as recently summarized by Kallman (1989). Lines from the disk would be broadened because of the optical depth to scattering and by Doppler shifts due to disk rotation (Kallman \& White 1989), by $\Delta E / E \approx 0.1$ is allowed by the SSS data. (Broadening of this order is also not ruled out by the OGS observations of the sources similar to X0614+091; Vrtilek et al. 1991.) The accretion stream's interaction with the outer disk could increase the amount of relatively low ionization gas that is subject to photoionization. Winds are more likely for the sources with luminosities approaching the Eddington limit (Melia, Zylstra, \& Fryxell 1991). The star is partially protected from the X-ray flux by the flaring disk. But contributions from a wind or a companion have not been carefully considered in the context of the presence of a corona.

The gas evaporated from the disk by the central source should have $k T_{c} \approx 1.25 \mathrm{keV}$ (Shakura \& Sunyaev 1976) and the emissivities would be $10^{-25}-10^{-23}$ ergs $\mathrm{cm}^{3} \mathrm{~s}^{-1}$, depending on the amount of photoionization (Kallman 1989; Kallman \&
McCray 1982), for the $\mathrm{O}$ and $\mathrm{Fe}$ lines that could contribute to the observed feature. Then the emission measure $E M=n_{e}^{2} V$ of the emitting region for a component of the observed feature is $\left(10^{58}-10^{60}\right)(d / 5 \mathrm{kpc})^{2} / n \mathrm{~cm}^{-3}$, where $n$ is the number of lines contributing, in the range $1-5$. This is to be compared with the $10^{58}-10^{59} \mathrm{~cm}^{-3}$ of brighter LMXB (Kallman 1989). The luminosity of X0614 + 09 was about $0.1(d / 5 \mathrm{kpc})^{2}$ times that of Sco $\mathrm{X}-1$ or $\mathrm{Cyg} \mathrm{X}-2$. The emission measures required to produce the X-ray lines around $0.8 \mathrm{keV}$ are in a ratio of about $1 / n(d / 5$ $\mathrm{kpc})^{2}$.

For an ionized corona in hydrostatic equilibrium (White and Holt 1982; Kallman \& White 1989; Kallman et al. 1989) the total coronal emission measure outside the radius $R_{7} \approx$ $10^{7} \mathrm{~cm}$ would be $1.1 \times 10^{60} f^{2} R_{7}^{-1 / 2} \Xi^{-2}\left(L / 7 \times 10^{36}\right)^{2} \mathrm{~cm}^{-3}$ (assuming a 1.4 solar mass neutron star and $k T_{c}=1.25 \mathrm{keV}$ ), with $f$ representing the effect of the glancing angle at which the flux is incident on the disk. For $f \approx 0.1$ and $\Xi \approx 10$, as generally assumed, the estimated emission measure is $10^{56} \mathrm{~cm}^{-3}$, falling short of the requirement by more than an order of magnitude. 
Furthermore, the part of the corona close to the source would be too highly ionized to be the source of lines below $1 \mathrm{keV}$. Thus the observed flux is not easily accounted for. Explaining line emission as strong as implied by the X0614 + 091 observations requires a better estimate of the coronal contribution or additional contributions.

The luminosity is sufficiently low that in the simple model the corona should not be optically thick, although the outer disk still would be shielded from the source. For more luminous low-mass X-ray binaries, optical depth effects may limit the coronal emission measures for low-energy lines (Kallman 1989; Kallman \& White 1989) and explain why they are not much brighter than X0614 + 091 .

Models of coronae which consider radiative transfer are being developed (Ostriker, McKee, \& Klein 1991; Ko \& Kallman 1992; Raymond 1993). They find self-consistent temperature distributions above the disk. Intermediate temperature zones in the transition from the optically thick disk to a corona at a Compton radiation temperature dominate the production of UV and soft X-ray lines. Raymond (1993) found that the flux of an $\mathrm{O}$ viII line was only $7 \times 10^{33} \mathrm{ergs} \mathrm{s}^{-1}$ for a $10^{38}$ ergs s$^{-1}$ central source and in general that the predicted line fluxes are 10 times less than reported in the Einstein OGS spectra.

An absorption edge consistent with the $\mathrm{K}$ edge of $\mathrm{O}$ VIII at $0.87 \mathrm{keV}$ was significant at the level of $99.9 \%$ in fitting the
October SSS + MPC data. An edge of optical depth $\tau_{a}$ for ionization of $O$ vIII, with $\sigma_{0}=3.4 \times 10^{-19} \mathrm{~cm}^{2}$ (Daltabuit \& Cox 1972) and $X_{0}=6.76 \times 10^{-4}$ for the abundance of oxygen implies a hydrogen column of $0.6 \times 10^{21}\left(\tau_{a} / 0.14\right) / X_{\mathrm{VIII}}$, where $X_{\mathrm{VIII}}$ is the fraction of the oxygen in the ionization state. This is a small fraction of the vertical column in the optically thin corona model, except for large radii in the disk. (We are probably not looking at the central source through a long path length near the base of the corona.) Considering radiative transfer effects (Kallman \& McCray 1982), oxygen should begin to recombine in the outer reaches of the corona, near the outer rim of the accretion disk, as far as $5 \times 10^{10} \mathrm{~cm}$ from the source. There would be a collar-like region where the ionization would be low enough to give rise to an absorption edge.

More quantitative measurements of lines and more exact calculations for the situations of interest are needed. The luminosity dependence of the line emission of LMXB should be investigated. The soft X-ray line emission from X0614+091 implies that the accretion disks of the moderate luminosity sources give rise to emission of coronal lines. It will be possible to use the soft X-ray emission and possibly absorption features in less luminous sources to measure properties of the disks and associated coronae.

We thank Dr. Timothy Kallman for helpful discussions.
Arnaud, K., \& Vrtilek, S. D. 1989, HEAO Newsletter, 1, 7

Bradt, H. V. D., \& McClintock, J. E. 1983, ARA\&A, 21, 13

Brandt, S., Castro-Tirado, A. J., Lund, N., Dremin, V., Lapshov, I., \& Sunyaev, R. 1992, A\&A, 262, L15

Christian, D. J. 1993, Ph.D. thesis, Univ. Maryland

Christian, D. J., Swank, J. H., Szymkowiak, A. E., \& White, N. E. 1992, Legacy, 1,38

Daltabuit, E., \& Cox, D. P. 1972, ApJ, 177, 855

Davidsen, A., Malina, R., Smith, H., Spinrad, H., Margon, B., Mason, K., Hawkins, F., \& Sanford, P. 1974, ApJ, 193, L25

Gaillardetz, R., Bjorkholm, P., Mastronardi, R., Vanderhill, M., \& Howland, D. 1978, IEEE Trans. NS, 25, 437

Giacconi, R., et al. 1979, ApJ, 230, 540

Holt, S. S., White, N. E., Becker, R. H., Boldt, E. A., Mushotzky, R. F., Serlemitsos, P. J., \& Smith, B. W. 1979, ApJ, 234, L65

Joyce, R. M., Becker, R. H., Birsa, F. B., Holt, S. S., \& Noordzy, M. P. 1978, IEEE Trans. NS, 25, 453

Kahn, S. M., Seward, F. D., \& Chlebowski, T. 1984, ApJ, 283, 286

Kallman, T. R. 1989, in Proc. 23rd ESLAB Symp. on Two Topics in X-Ray Astronomy, ed. J. Hunt \& B. Battrick (ESP SP-206), 157

Kallman, T. R., \& McCray, R. A. 1982, ApJS, 50, 263

Kallman, T. R., Vrtilek, S. D., \& Kahn, S. M. 1989, ApJ, 345, 498

Kallman, T. R. \& White, N. E. 1989, ApJ, 341,955

Ko, Y.-K., \& Kallman, T. K. 1992, in Proc. 10th Internat. Colloq. on UV and in-Ray Spectroscopy of Astrophysical and Laboratory Plasmas, ed. S. Kahn, in press

\section{REFERENCES}

Liedahl, D., Kahn, S. M., Goltstein, W., \& Osterheld, A. 1989, in Proc. 23rd ESLAB Symp. On Two Topics in X-Ray Astronomy, ed. J. Hunt \& B. Battrick (ESP SP-206), 505 .1990, ApJ, 350, 137

Machin, G., et al. 1990, MNRAS, 247, 205

Mason, K. O., Charles, P. A., White, N. E., Culhane, J. L., Sanford, P. W., \& Strong, K. T. 1976, MNRAS, 177, 513

Marshall, N., \& Millit, J. M. 1981, Nature, 293, 379

Melia, F., Zylstra, G. J., \& Fryxell, B. 1991, ApJ, 377, L101

Mitsuda, K., et al. 1984, PASJ, 36, 741

Morris, R., \& McCammon, D. 1983, ApJ, 270, 119

Ostriker, E. C., McKee, C. F., \& Klein, R. I. 1991, ApJ, 377, 593

Raymond, J. C. 1993, ApJ, 412, 267

Shakura, N. I., \& Sunyaev, R. A. 1976, A\&A, 24, 337

Shull, J. M. 1979, ApJ, 234, 761

Swank, J. H., Becker, R. H., Boldt, E. A., Holt, S. S., \& Serlemitsos, P. J. 1978 MNRAS, 182, 349

van Paradijs, J., Dotani, T., Tanaka, Y., \& Tsuru, T. 1990, PASJ, 42, 633

Vrtilek, S. D., Helfand, D. J., Halpern, J. H., Kahn, S. M., \& Seward, F. D. 1986a, ApJ, 308, 644

Vrtilek, S. D., Kahn, S. M., Grindlay, J. E., Helfand, D. J., \& Seward, F. D. 1986b, ApJ, 307, 698

Vrtilek, S. D., McClintock, J. E., Seward, F. D., Kahn, S. M., \& Wargelin, B. J. 1991, ApJS, 76, 1127

White, N. E., \& Holt, S. S. 1982, ApJ, 257, 318

White, N. E., Peacock, A., \& Taylor, B. G. 1985, ApJ, 296, 475 